\title{
Special issue: Computational models of natural argument
}

\author{
Floriana Grasso $^{\mathrm{a}, *}$, Floris Bex ${ }^{\mathrm{b}}$ and Nancy Green ${ }^{\mathrm{c}}$ \\ ${ }^{a}$ University of Liverpool, $U K$ \\ ${ }^{\mathrm{b}}$ Utrecht University, The Netherlands \\ ${ }^{\mathrm{c}}$ University of North Carolina Greensboro, USA
}

The international workshop on Computational Models of Natural Argument (CMNA) is the longest standing venue focussed on Argument and Computation, with its inception in 2001. During the lifespan of CMNA, many other international venues have been constituted (see Table 1), most notably the ArgMAS workshops focusing on Agents since 2004, the biennial COMMA conference series focusing on computational models and tools since 2006, the TAFA workshop series focusing on formal models since 2011, the Argument Mining workshops since 2014, and the very new, biennial European Conference of Argumentation, ECA, in 2015, as well as a number of standalone events.

Notwithstanding the proliferation of events, CMNA maintains a distinct character for two special reasons. First, its theme: centering on natural argument, in the way in which argumentation is used

Table 1

Timeline of argument and computation events

\begin{tabular}{lll}
\hline 2016 & CMNA XVI - New York (IJCAI) & \\
2015 & CMNA XV - Bertinoro (PRIMA) & 1st ECA \\
2014 & CMNA XIV - Krakow (JURIX) & 1st Argument Mining Workshop \\
2013 & CMNA XIII - Rome (ICAIL) & \\
2012 & CMNA XII - Montpellier (ECAI) & \\
2011 & CMNA XI - San Francisco (AAAI) & 1st TAFA \\
2010 & CMNA X - Lisbon (ECAI) & \\
2009 & CMNA IX - Pasadena (IJCAI) & 1st Argument \& Computation Journal \\
2008 & CMNA VIII - Patras (ECAI) & \\
2007 & CMNA VII - Hyderabad (IJCAI) & \\
2006 & CMNA VI - Riva del Garda (ECAI) & 1st COMMA \\
2005 & CMNA V - Edinburgh (IJCAI) & \\
2004 & CMNA IV - Valencia (ECAI) & 1st ArgMAS \\
2003 & CMNA III - Acapulco (IJCAI) & \\
2002 & CMNA II - Lyon (ECAI) & \\
2001 & CMNA I - San Francisco (ICCS) & \\
\hline
\end{tabular}

*Corresponding author. E-mail: floriana@liverpool.ac.uk. 
in context, as well as the modelling "with" argument, not only "of" argument, continuing to provide inspiration and research challenges right across the fields of AI and cognitive science. Second, its nature: CMNA is still a venue for scholarly discussion and informal presentations, rather than a primarily publication oriented venue, uniquely positioned in the current climate.

The workshop focuses on the issue of modelling "natural" argumentation, where natural can mean different things in different contexts. Naturalness may involve the use of means which are more visual than linguistic to illustrate a point, such as graphics or multimedia, or the use of more sophisticated rhetorical devices, interacting at various layers of abstraction, or the exploitation of "extra-rational" characteristics of the audience, taking into account emotions and affective factors, or simply the way in which argumentation "happens" in various contexts. In particular, throughout the years, CMNA has seen contributions on ontological aspects and cognitive issues, the linguistic characteristics of natural argumentation, the generation of natural argument, corpus argumentation results and techniques, models of arguments in various contexts, from the legal to the medical to the pedagogical or the financial, discussions on rhetoric and affect, the role of emotions in argumentation, and the list goes on.

While, as said before, no formal publication is envisaged as a final aim of the workshops, the event has produced a number of archival output throughout the years, such as a special issue of the International Journal of Intelligent Systems, and volumes with revised paper collections. The present issue is the latest of this output, and presents a selection of papers from more recent editions, all revised and extended.

The papers in this issue demonstrate the broad and interdisciplinary character of CMNA. There is a paper about an innovative, argument-based app for healthy living (Lindgren et al. "An activity-centric argumentation framework for assistive technology aimed at improving health"), more formal theoretical work based on Pollock's epistemological and cognitively inspired type of argumentation (Wei \& Prakken's "An analysis of critical-link semantics with variable degrees of justification"), and a more semi-formal approach to distinguishing argument and explanation (Bex and Walton's "Combining explanation and argumentation in dialogue"). Furthermore, two papers investigate the connections between arguments in natural language and more formal, structured arguments (Wyner et al.'s "Working on the argument pipeline: through flow issues between natural language argument, instantiated arguments, and argumentation frameworks" and Budzynska et al.'s "Theoretical foundations for illocutionary structure parsing").

The diversity of topics in this issue testifies to the number of communities reached out by CMNA: the decision to maintain it as a peripatetic venue, co-hosted by different conferences, ensures CMNA is able to expose argumentation theory and practice to different sub-communities of AI, and in turn other sister disciplines. This has helped create a community of scholars who supports the workshop in its various editions as a dedicated, committed and ever expanding Programme Committee, in turn securing a healthy and interesting workshop environment for the future.

The CMNA steering committee Floriana Grasso University of Liverpool Floris Bex

Utrecht University Nancy Green 